\title{
La molécule d'activation CD26 des cellules T est nécessaire à l'entrée du VIH dans les cellules $\mathrm{CD4}^{+}$
}

Le virus de l'immunodéficience humaine (VIH) infecte les lymphocytes $\mathrm{T}$, monocytes et macrophages en se fixant à leur récepteur connu, la molécule CD 4 ; celle-ci est essentielle à la fixation des particules virales sur les cellules cibles permissives, mais non suffisante pour une entrée virale et une infection efficace. Les travaux de plusieurs équipes ont suggéré l'existence d'un co-récepteur spécifique de l'espèce qui serait impliqué dans l'entrée du VIH. D'autres études ont suggéré, sans apporter de preuves directes, l'existence d'un clivage de la boucle V3 de la glycoprotéine d'enveloppe externe du VIH, la gpl20, au cours du processus d'entrée du virus. Il est maintenant bien établi que la boucle V3 joue un rôle critique dans l'infection du VIH; en effet, des anticorps neutralisants dirigés contre cette boucle, et des mutations ponctuelles dans ce domaine de la gpl20, modifient le tropisme cellulaire et affectent l'infectivité des particules virales. L'entrée du VIH implique donc une interaction entre la boucle V3 et un composant cellulaire probablement doté d'une activité protéolytique [1].

Sur la base de cette hypothèse, notre stratégie [2] fut donc de sélectionner un candidat qui pourrait interagir avec la boucle V3. Parmi les différentes protéases connues et présentes à la surface des lymphocytes $T$, une seule avait la spécificité requise pour les séquences conservées de la boucle V3. Cette protéase est la dipeptidyl peptidase IV (DPPIV), également connue sous le nom de CD26.

\section{CD26/DPP-IV}

La protéine (.D26/DPP-IV [3] est considérée comme une exopeptidase qui clive préférentiellement les dipeptides dans la partie aminoterminale des substrats polypeptidiques, dès lors que le deuxième résidu est une proline: soit une séquence de type X-P-polypeptide, $\mathrm{P}$ étant un résidu de proline et $\mathrm{X}$ un acide aminé $N$-terminal libre, de préférence un résidu de glycine, alanine, lysine ou arginine (respectivement $G, A, K$ ou $R$ ). La DPP-IV peut se lier au collagène vraisemblablement par ses motifs répétés XProline-(ilycine, mais cette liaison n'affecte pas son activité enzymatique [4], suggérant ainsi l'existence de sites de fixation et de sites catalytiques localisés dans différents domaines de la DPP-IV. Cette enzyme est exprimée dans plusieurs types cellulaires, notamment les lymphocytes $\mathrm{T}$, les macrophages et les cellules folliculaires dendritiques. CD26/DPP-IV est considérée comme un antigène d'activation des lymphocytes T. En effet l'expression de la DPP-IV est faible dans les lymphocytes $\mathrm{T}$ au repos, mais elle augmente considérablement après activation. La DPP-IV semble être un relais obligatoire dans le signal physiologique d'activation cellulaire du lymphocyte $T$.

\section{Suivi du processus d'entrée du VIH}

L' entrée des particules du VIH dans les cellules est suivie par la mesure de la concentration intracellulaire de la protéine majeure de la nucléocapside du virus (p24/p25), après élimination par la trypsine des virions adsorbés à la surface cellulaire. I a pénétration du virus nécessite une incubation à $37^{\circ} \mathrm{C}$; elle est bloquée par des molécules polyanioniques [5] telles que l'héparine, le sulfate de dextran, ou poly(A). poly(U), un ARN bicaténaire synthétique. L'utilisation habituelle de ces inhibiteurs de l'entrée est indispensable pour démontrer l'efficacité du traitement par la trypsine car ils n'affectent pas l'accrochage de la gpl 20 au CD4 mais inhibent à plus de $95 \%$ l'entrée du virus. Après l'étape du traitement à la trypsine, les cellules peuvent être mises en culture afin de mesurer la production virale. Dans ces conditions expérimentales, nous observons une corrélation étroite entre la quantité de virus entrée après $1 \mathrm{~h}$ d'infection et la quantité de virus produite après trois jours d'infection.

La molécule CD26 est impliquée dans l'entrée du virus [2]

1. L'analyse des séquences peptidiques de la boucle V3 des isolats VIH-1, VIH-2 et SIV montre une conservation de motifs qui correspondent à la spécificité enzymatique de la DPP-IV. La boucle V3, caractérisée comme le domaine principal de neutralisation, est une partie variable de la gp120; cette variabilité permet au virus d'échapper à la réponse aux anticorps neutralisants. Malgré cela, il existe quelques motifs conservés dans plus de $90 \%$ des séquences des boucles V3 connues. Ces motifs dipeptidiques comportent une proline: GP dans le cas des

(

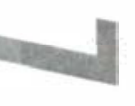


$\mathrm{VIH}-1$ consensus (Amérique du Nord et Europe; 525 isolats)

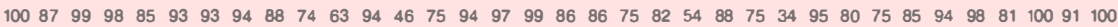
CTRPNNNTRKS I I GPGRAFYTTGE I I GD I RQAHC

$\mathrm{VIH}-2$ consensus (25 isolats)

$1009210010096100100929659968585 \quad 778910010070559610096929281100448910085100100100100$ CKRPGNKTVVP I T L MSGLVFHSQP I NKRPRQAWC

SIV ( 8 isolats)

1001001001001001001001001001001001001001001008888100100100100100100887510050888888100100100100 CRRPGNKTVLPVTIMSGLVFHSQP I NDRPKQAWC

Figure 1. Les consensus de la séquence de la boucle V3 pour VIH-1, VIH-2 et SIV. Les motifs conservés RP, KP et GP sont en caractères rouges, les nombres au-dessus de chaque acide aminé indiquent le pourcentage de conservation.

VIH-1, RP dans la partie N-terminale de la boucle V3 pour tous les isolats VIH-1, VIH-2, SIV et RP/KP dans la partie C-terminale des isolats VIH-2 et SIV ([6] et figure 1).

2. Des peptides correspondant à la boucle V3 (environ 35 acides aminés) inhibent l'entrée du VIH dans les cellules $\mathrm{T}$ (.D) $4^{+}$, probablement par compétition avec la gpl20 des particules virales. Cet effet est spécifique puisque d'autres peptides de la gp120 n'inhibent pas de façon significative l'entrée du VIH dans les cellules. Comme la boucle V3 n'est pas directement impliquée dans la liaison de la gp120 au CD4, ces résultats suggèrent que les peptides de la boucle V3 inhibent l'entrée du virus en interagissant avec une protéine de surface autre que le (i) 4.

3. Le tripeptide IPI, un inhibiteur spécifique de l'activité protéolytique de la IDPP-IV, bloque l'entrée du virus, alors que des inhibiteurs spécifiques de la plupart des endopeptidases et des exopeptidases connues hormis la DPP-IV n'ont aucun effet sur l'entrée du VIH.

4. Des peptides synthétiques renfermant les motifs GP, KP ou RP, les motifs conservés de la boucle V3, inhibent l'entrée du virus ainsi que l'activité enzymatique de la DPP-IV. Différents tripeptides témoins, utilisés aux mêmes concentrations, n'ont aucune action sur l'entrée du virus. 5. Des anticorps monoclonaux spécifiques de (.I)26 inhibent l'entrée du virus, comme des anticorps monoclonaux spécifiques de la boucle V3 ou de la molécule C.D4.

6. Les tripeptides IPI et KPR, et l'anticorps anti-CD26 bloquent l'infection des cellules par l'isolat VIH-1 I AI ou par l'isolat VIH-2 EHO. Sachant que les boucles V3 de ces deux virus ne sont pas apparentées, ces observations démontrent que l'implication de CD26 pour l'entrée virale est un phénomène général pour les différents isolats et types du VIH.

7. Les cellules humaines (HeLa) et murines (NIH 3T3) CD4- expriment CD26 à leur surface mais l'expression du CD4 humain dans ces cellules ne rend permissives à l'infection par le VIH que les cellules humaines. En effet, bien que les particules du VIH s'accrochent aux cellules NIH 3T3 exprimant la molécule CD4 humaine, l'entrée virale ne se produit pas. En conséquence, si notre hypothèse est fondée, la molécule CD26 devrait être spécifique de l'espèce, comme c'est le cas pour la molécule CD4. A cet égard, nous avons démontré que l'effet des inhibiteurs tripeptidiques, tels que IPI et KPR, sur l'activité de la DPPIV humaine est au moins cent fois plus élevé que sur l'activité de la DPP-IV murine. Cela démontre la différence de spécificité entre les enzymes des deux espèces.

8. La co-expression de (CI)4 et CD26 humains dans les cellules NIH-3T3 rend ces cellules murines permissi- ves à l'infection par le VIH-l et le VIH-2. L'addition d'inhibiteurs de l'entrée, tels que le tripeptide IPI ou l'héparine, bloque l'infection des cellules murines exprimant les molécules CD4 et CD26 humaines. L'addition d'AZT au cours de l'infection des cellules transfectées bloque aussi l'infection virale, ce qui démontre que la réplication du VIH a eu lieu dans les cellules infectées.

\section{Mécanisme d'entrée}

Pour expliquer les mécanismes d'action des inhibiteurs tripeptidiques au niveau de l'entrée virale, nous avons étudié la fixation de molécules de gp120 marquées à l'iode sur des cellules (.EM. Cette fixation est spécifique car elle est bloquée par des anticorps anti-CD4 ou anti-gp120. En revanche, les inhibiteurs tripeptidiques IPI et KPR, à des concentrations qui bloquent l'entrée virale à plus de $95 \%$, n'ont aucun effet sur la fixation de la gpl20 aux cellules (.D4+. Ces observations démontrent que les cibles touchées par ces inhibiteurs peptidiques se trouvent à une étape ultérieure de la fixation de la gp120 au CI)4, c'est-à-dire, CD26. Au cours de la fixation de la particule virale au CI) 4, un changement conf ormationnel dans le complexe gpl20/gp4l (la glycoprotéine transmembranaire) pourrait démasquer la boucle V3, la rendant accessible au CD26. Par la suite, l'interaction du domaine $\mathrm{N}$ terminal hydrophobe de la gp4l avec la membrane cellulaire entraine le fusionnement des membranes virale et cellulaire, une étape essentielle pour l'introduction du core viral dans les cellules.

\section{Perspectives}

Bien que CD26 soit impliqué dans l'entrée virale, la nécessité de son activité catalytique n'a pu être démontrée de façon directe. L'inhibition de l'entrée du VIH par des inhibiteurs de l'activité enzymatique de DPP-IV suggère que le site catalytique et/ou le site de fixation pourraient être impliqués. En outre, la molécule CD26 servirait de corécepteur de la molécule $\mathrm{CD} 4$. 
Le rôle de CD26 dans l'entrée et l'infection par le VIH est en accord avec d'autres études in vivo, démontrant une diminution sélective des lymphocytes $\mathrm{T} \mathrm{CD}^{+}, \mathrm{CD}^{2} 6^{+}$chez les patients atteints du SIDA [7].

Le développement du SIDA chez les individus séropositifs est corrélé à la charge virale, c'est-à-dire à l'infection des lymphocytes $\mathrm{CD}^{+}$dans le sang et les tissus lymphoïdes [8]. Les connaissances actuelles sont insuffisantes pour le développement de médicaments qui pourraient éliminer le $\mathrm{VIH}$, mais on devrait pouvoir développer des agents antiviraux agissant aux différentes étapes de l'infection par le VIH. La démonstration que CD26/DPP-IV est un corécepteur du VIH offre la possibilité de développer de nouveaux inhibiteurs de l'infection. L'inhibition de l'entrée des virus du type VIH-l et VIH-2 par des peptides tels que IPI démontre que des agents antiviraux de structure simple, peptidiques ou non, pourraient être utilisés dans le cadre de protocoles thérapeutiques, afin de diminuer la charge virale chez les patients séropositifs ou atteints de SIDA. Bien évidemment, pour atteindre ce but, il est important de développer des inhibiteurs efficaces à des concentrations de l'ordre de la nanomole/l. De plus, les motifs dipeptidiques fortement conservés de la boucle V3 des VIH-l et VIH-2 peuvent être considérés comme des cibles potentielles pour le développement de vaccins contre les différents types et sous-types de VIH.

C.C.

A.G.H.

1. Moore JP, Nara PI. The role of the V 3 loop of gpl20 in HIV infection. AIIJS 1991; 5: $21-33$.

2. Callebaut C, Krust B, Jacotot E, Hovanes- sian AG. T cell activation antigen, CI)26 as a cofactor for entry of HIV in (.D) $4^{+}$cells. Science 1993; 262: 2045-50.

3. Barclay AN, Birkeland MI., Brown MH, Beyers AI), Davis S], Somoza (., Williams AF. The leucocyte antigen. In: Facts book. I.ondon: Harcourt Brace Jovanich, 1993.

4. Hanski C, Huhle T, Gossrau R, Reutter W. Direct evidence for the binding of rat liver DPP-IV to collagen in vitro. Exp Cell Res 1988 . 178: 64-72.

5. Krust B, Callebaut C, Hovanessian AC; Inhibition of entry into cells by poly(A).poly(U). AII)S Res Hum Retrrir 1993; 9 : 1081-4.

6. Myers (;, Berzof sky JA, Korber BRFS, Pavlakis (;N. Human retroviruses and AII)S. I.OS Alamos, USA : I.os Alamos National I.aboratory, 1992.

7. Vanham (;, Kestens I., De Meester I, V'ingerhoets J, Penne (;, Vanhoof (;, Scharpé S, Heyligen H, Bosmans E, Ceuppens JI., (;igase P. Decreased expression of the memory marker C.I)26 on both (:D) $4^{+}$and (.D $8^{+} \mathrm{T}$ lymphocytes of HIV'-inf ected subjects. / A/ISS $1993 ; 6$ : 749-57.

8. Pantaleo (', Craziosi C, Demarest JF, Butini I., Montroni M, Fox CH, Orenstein JM, Kotler IDP, Fauci AS. HIV' infection is active and progressive in lymphoid tissue during the clinically latent stage of disease. Nature 1993; 362: 355-8.

\section{口U BRÈVES}

पa réponse immunitaire des souris déficientes en interleukine 2. La stimulation des lymphocytes $\mathrm{T}$ dépend de deux signaux transmis par le récepteur de l'antigène et par les molécules d'activation CD28 et CTIA-4. Cette cosignalisation augmente très fortement la production d'IL2, essentielle pour la prolifération des lymphocytes $\mathrm{T}$ in vitro. L'IL2 est principalement produite par les lymphocytes T $\mathrm{CD}^{+}$auxiliaires activés et est considérée comme une hormone de croissance indispensable au développement d'une réponse immunitaire. Ce concept vient pourtant d'être remis en question par l'étude des souris rendues déficientes en IL2 par recombinaison homologue. La fonction cytotoxique des lymphocytes $\mathrm{T} \mathrm{CD}^{+}$ des souris II.2- / $^{-}$a été étudiée en infectant les souris par les virus de la vaccine ou de la chorioméningite lymphocytaire (LCMV), car la réponse cytotoxique est un facteur clef dans la lutte contre ces infections virales[1]. Pour analyser la réponse humorale, l'infection par le virus de la stomatite vésiculeuse (VSV) a été utilisée car c'est un modèle pour lequel la coopération B-T auxiliaires joue un rôle primordial. Les réponses cytotoxiques, primaires et secondaires, spécifiques du virus de la vaccine et du LCMV ne sont pas significativement altérées chez les souris jeunes IL2- $/$ par rapport aux souris $\mathrm{IL}^{+} /-$ou $\mathrm{IL}^{+} /+$ du même âge. Chez ces souris (4 à 6 semaines) l'absence d'IL2 pourrait donc être compensée efficacement par d'autres cytokines. Il faut noter cependant que des souris plus agées (plus de huit semaines) meurent d'une maladie aux multiples syndromes et ne développent pas de réponses cytotoxiques spécifiques. La réponse IgM dirigée contre le VSV, indépendante des lymphocytes $\mathrm{T}$ auxiliaires, est identique chez les souris IL2 $/^{-}$et IL2 $/^{-}$et montre que les lymphocytes $B$ peuvent fonctionner normalement malgré l'absence d'IL2. En revanche, la commutation isotypique, dépendante des lymphocytes T auxiliaires $\mathrm{CD}^{+}{ }^{+}$, est retardée chez les souris IL2- ${ }^{-}$. Cela indique que la fonction $\mathrm{T}$ auxiliaire, bien que touchée par l'absence d'IL2, reste quand même efficace. L'activité NK (natural killer) est inductible, mais fortement réduite, chez les souris mutantes infectées par le LCMV. La prolifération des cellules NK paraît donc bien dépendante de la présence de l'Il.2, mais l'inductibilité de la fonction serait plutôt sous le contrôle d'autres cytokines telles que les interférons $\alpha$ et $\beta$. A la lecture de ce travail, il semble que l'importance donnée à l'IL2 dans le développement et la régulation d'une réponse immunitaire devrait être considérée avec plus de modération. Dans le cadre d'un système adaptatif et redondant comme le système immunitaire, il est tout à fait concevable que l'absence d'une lymphokine puisse être compensée par l'action combinée de plusieurs autres.

[1. Kundig TM, et al. Science 1993; 262: 1059-61.] 
Phénotype femelle malgré le génotype mâle et l'intégrité du locus Sry. La mutation murine Sxr ${ }^{a}$ présente la particularité de porter au niveau de la région pseudoautosomique du chromosome $\mathrm{Y}$ une duplication partielle du bras court de ce chromosome, comprenant notamment le gène Sry qui est responsable de la détermination sexuelle mâle. La région pseudoautosomique, également présente à l'extrémité du bras court du chromosome X, est la seule zone d'appariement et d'échanges entre les deux chromosomes lors de la méiose masculine. La région dupliquée peut donc être transférée sur l'X par recombinaison au cours d'une méiose. Les mâles ainsi obtenus (Xsxri/Y) donnent une descendance de souriceaux dont certains peuvent être porteurs de délétions variables $\mathrm{du}$ chromosome $\mathrm{Y}$ par recombinaison inégale. C'est ainsi qu'une équipe anglaise [1] a obtenu, sur 450 descendants, trois femelles fertiles de caryotype XY. Chez ces trois animaux, aucun remaniement moléculaire n'est détecté sur un locus de $36 \mathrm{~kb}$ encadrant le gène Sry et incluant notamment les $14 \mathrm{~kb}$ qui, introduits par transgénèse dans le génome d'une souris femelle, suffisent à inverser le sexe en mâle. Ainsi, le développement génital de ces trois souris est resté orienté vers le sexe femelle malgré l'intégrité du locus Sry. C'est en réalité à distance de ce locus que des délétions, portées par le bras court du même chromosome $Y$ mais plus proches du centromère, ont été mises en évidence chez ces trois souris, dans une région d'ADN nommée Sxl. L'expression du gène Sry a été étudiée chez les descendants d'une d'entre elles, à 11,5 jours de développement embryonnaire, et a été trouvée diminuée ou abolie. Ces résultats ne remettent donc pas en question l'importance de Sry dans la détermination du sexe mâle. Ils suggèrent simplement le rôle de séquences adjacentes dans la modulation de l'expression de ce gène. centromériques de l'Y à proximité du locus Sry pourrait, en effet, avoir favorisé l'extinction de ce dernier. Il reste que ce type de remaniement chromosomique permet d'obtenir, par croisement, une variété de mutations dont le phénotype peut être très riche d'enseignements. Rappelons enfin qu'une délétion extérieure au gène Sry mais proche de celui-ci (à 1,7 kb en amont du site d'initiation de la transcription) avait déjà été décrite chez une femme $(46, X Y)$ porteuse d'une dysgénésie gonadique [2].

[1. Capel B, et al. Nature Genet 1993 ; 5 : 301-7.]

[2. McElreavy K, et al. Proc Natl Acad Sci USA 1992; 89 : 11016-20.]

L'interleukine- 12 restaure, in vitro, les réponses immunes cellulaires, spécifiques du VIH. Les mononucléaires périphériques de nombreux sujets séropositifs pour le VIH, mais asymptomatiques, ne répondent pas à une stimulation par des virus ou des peptides synthétiques d'enveloppe virale, par la prolifération normale des cellules $T$ et la sécrétion d'IL2. En effet, au cours de la progression vers le SIDA des malades infectés par le VIH, on observe, en réponse à une agression immunologique, une commutation du profil de sécrétion des cytokines de type T helper 1 (IL2 élevée, IL4 et IL.10 basses) vers le type T helper 2 (IL2 basse, IL4 et IL10 élevées) [1]. Ce changement dans le profil des cytokines a une valeur prédictive quant au déclin du nombre des lymphocytes CD4 et au délai d'installation d'un SIDA. Les anticorps anti Il4 et anti Ill 0 sont capables d'inverser, in vitro, cette commutation TH1-TH2 et de restaurer, in vitro, la production d'IL2 par les mononucléaires périphériques. De plus, on a montré récemment que IL12 était capable de court-circuiter l'effet inhibiteur d'ILl0 sur l'induction de la fonction $\mathrm{TH} 1$; peut-elle aussi reconstituer la fonction $\mathrm{TH} 1$ des mononucléaires des patients séropositifs pour le VIH? IL12 stimule normalement l'activité des cellules NK et des lymphocytes cytotoxiques, la prolifération des lymphocytes $\mathrm{T}$ et la production d'interférón $\gamma$; est-elle produite en quantité insuffisante au cours de l'infection par le VIH? L'équipe de Shearer, du NIH à Bethesda (MD, USA), vient de montrer que IL12 est capable de restaurer, in vitro, toutes les réponses immunologiques normales au virus de la grippe ou à des peptides synthétiques de l'enveloppe du VIH, de cellules infectées par le VIH : prolifération, sécrétion d'IL2, production d'interféron $\gamma[3]$. Les cellules de donneurs témoins, dans les mêmes conditions de stimulation, ont une réponse qui n'est pas augmentée par l'addition d'IL12. IL12 est donc sans doute en quantité limitante chez les malades $\mathrm{VIH}^{+}$, ce qui suggère l'utilité potentielle de cette cytokine pour restaurer in vivo les fonctions immunologiques chez ces malades. Mais il faudrait d'abord connaître un peu mieux cette cytokine et, en particulier, sa toxicité éventuelle chez l'homme avant de pouvoir procéder à de premiers essais in vivo.

[1. Clerici M, et al. J Clin Invest 1993 ; 91 : 759-65.]

[2. Lucey DR, et al. J Infect D)is 1991 ; $164: 631-7$.

[3. Clerici M, et al. Science 1993; 262 : 1721-4.]

Le déficit en lipase acide. La lipase acide (ou hydrolase des esters acides du cholestérol) est une enzyme des lysosomes qui joue un rôle important dans l'équilibre des lipoprotéines. Son déficit est cause de deux entités cliniques, à hérédité autosomique récessive: la maladie de Wolman, de haute gravité, en 


\section{BREVES}

général mortelle dès la première année, et une maladie de surcharge en esters du cholestérol, dont le symptôme clinique principal est une hépatosplénomégalie. Le diagnostic se fonde sur l'élévation des esters du cholestérol et des triglycérides, et sur la chute de l'activité de la lipase acide dans les leucocytes et les fibroblastes en culture. On sait depuis près de quinze ans [1] que le gène de la lipase acide siège sur le chromosome 10; un ADNc a été cloné en 1991 [2] ; il s'étend sur 2,6 kb, et code pour une protéine de 378 acides aminés. Une équipe allemande [3] a pour la première fois tenté de définir une lésion moléculaire chez un garçon de 12 ans atteint de la forme modérée. Elle a trouvé sur l'ADNc une délétion en phase de 72 nucléotides, faisant disparaître les acides aminés 254-277. Mais l'analyse de l'ADN génomique a montré qu'il n'y avait pas de délétion, et qu'une mutation ponctuelle $\mathrm{G} \rightarrow \mathrm{A}$ dans le dernier nucléotide d'un exon de $72 \mathrm{pb}$ provoquait un épissage anormal avec élimination d'un exon. La connaissance de ce malade reste encore incomplète: bien que cette mutation soit à l'état hétérozygote (héritée de la mère), on ne détecte aucun messager de taille normale ; on est donc conduit à penser, sans preuve encore, que l'allèle hérité du père est nul et qu'il s'agit d'un hétérozygote composite, dont la nature reste à préciser. La délétion des acides aminés 254-277 ne se trouve pas dans la zone catalytique, et ne comporte pas de site de glycosylation. La protéine conserve probablement une activité résiduelle expliquant la relative bénignité du syndrome malgré l'absence du deuxième allèle. Il restera également à démontrer que dans la maladie de Wolman, beaucoup plus sévère, c'est le même gène qui est en cause.

[1. Nguyen VC, et al. Hum Genet $1980 ; 55$ : 375-83.]

[2. Anderson RA, Sando GN. I Biol Chem 1991; 266 : 22479-84.]

[3. Klima H, et al. J Clin Invest 1993; 92: 2713-8.]
Que deviennent les stocks de fer des thalassémiques après transplantation de moelle? Les malade homozygotes pour une $\beta$-thalassémie survivent grâce aux transfusions sanguines dont la complication majeure, à côté de l'immunisation et de la transmission des maladies virales, est la surcharge martiale. Le traitement chélateur du fer par injection parentérale de desferrioxamine, lorsqu'il est bien suivi, permet de retarder la survenue et de réduire la sévérité des atteintes organiques dues à cette surcharge mais ne permet pas de les éviter, et la biopsie de foie en est le meilleur indicateur. La transplantation de moelle est le seul moyen de guérir définitivement les malades atteints de thalassémie majeure. L'équipe de Lucarelli à Pesaro (Italie), l'initiatrice de la greffe de moelle dans cette indication, reste de très loin celle qui en a la plus grande expérience avec le meilleur taux de réussite. Une fois greffés, ces sujets deviennent des exthalassémiques, gardant les stigmates de leur maladie antérieure, en particulier la surcharge en fer. Lucarelli et al. rapportent leur étude sur le devenir des stocks de fer de malades greffés depuis 1981 [1]. Les malades sont divisés en trois catégories selon les facteurs de risque avant la transplantation que sont l'hépatomégalie, la fibrose portale hépatique et la qualité du traitement chélateur. Le premier groupe n'a pas de facteur de risque, le second en a un ou deux et le troisième a les trois. Dans les cing années qui suivent la transplantation, les malades du premier groupe font quasiment disparaittre leur surcharge : la ferritine sérique revient à la normale ainsi que la capacité de liaison du fer non lié. La biopsie hépatique montre une surcharge en fer discrète ou absente. Dans le deuxième groupe, dont $80 \%$ des malades avaient une surcharge hépatique modérée ou sévère avant transplantation, $60 \%$ des malades n'ont plus qu'une surcharge discrète ou absente après cing ans. En revanche, les malades du troisième groupe conservent pour la plupart une surcharge modérée ou sévère. La plus grande partie du fer est stockée dans le foie sous forme d'hémosidérine, séquestrée, ne participant pas à la production de radicaux libres, et est peu mobilisée par le traitement chélateur. Les risques de fibrose hépatique, de cirrhose et d'hépatome sont cependant accrus et ces malades commencent à être traités par saignée.

[1. Lucarelli G, et al. Lancet 1993 ; 342 : 1388-91.]

B7, activation lymphocytaire et transmission virale. La prolifération lymphocytaire est déclenchée en général par deux signaux: le premier signal provient de la reconnaissance, par le récepteur de l'antigène, des peptides présentés par les molécules du complexe majeur d'histocompatibilité (CMH) et le second signal est fourni par les récepteurs CD28 et CTLA-4 lors de leur interaction avec la molécule B7, exprimée par les cellules présentatrices. L'infection par le virus de l'immunodéficience humaine (VIH) passe par un état d'activation des lymphocytes T qui pourrait s'établir par l'intermédiaire des molécules CD28/CTLA-4 et B7. Cette éventualité a été testée à l'aide de cultures primaires de lymphocytes T CD4 de patients infectés par le VIH-1, activées par stimulation allogénique [1]. L'expression de plusieurs récepteurs associés à l'activation cellulaire a été étudiée à la surface des lymphocytes avec des anticorps monoclonaux. Après une stimulation allogénique répétée, l'expression de CD28 et de CD4 est fortement réduite à la surface des cellules T CD4 infectées alors que d'autres marqueurs restent stables (CD3, CMH-II, CD2, LFA-1). La production virale est accrue et le taux d'infection peut atteindre $50 \%$ à $90 \%$ selon les cultures. L'expression de CTIA-4 par les lignées infectées augmente rapidement mais transitoirement puisqu'elle redevient 
normale après sept jours de stimulation. B7 peut être exprimée par les lymphocytes $T$ et son niveau d'expression augmente lors de l'infection par HTLV-1. C'est aussi le cas lors de l'infection par le VIH. L'augmentation de l'expression de B7 est graduelle pendant les trois semaines de stimulation des lymphocytes T CD4 de donneurs séropositifs. Ce phénomène est également observé avec des lymphocytes de donneurs séronégatifs, mais après une stimulation beaucoup plus longue, de sept semaines. L'accumulation des ARNm de l'IL.2 est indépendante de l'infection virale mais nécessite l'intervention de B7. En revanche, $\mathrm{B} 7$ et ses récepteurs ne semblent pas contrôler la transcription virale. La présence du CMH-II et de $\mathrm{B} 7$ à la surface des lymphocytes T CD4 infectés rend ces cellules potentiellement capables de stimuler à leur tour d'autres cellules $\mathrm{T}$. De fait, les lymphocytes T CD4 de donneurs séronégatifs s'activent au contact des lignées $T$ infectées allogéniques, phénomène qui s'accompagne d'une production virale importante dans les cultures. L'interaction de B7 avec ces récepteurs interviendrait donc directement dans la transmission virale, des cellules stimulatrices infectées vers les cellules répondeuses non infectées. A la lecture de cet article, quelques idées viennent à l'esprit qui méritent d'être considérées avec soin. Si les lymphocytes $\mathrm{T}$ CD4 sont, dans ce travail, activés par une stimulation allogénique, un phénomène semblable pourrait se produire in vivo chez les patients séropositifs. Il faut postuler pour cela que les cellules infectées expriment à leur surface des molécules du CMH-II inhabituelles, reconnues par les lymphocytes $\mathrm{T}$ non infectés. Cela pourrait se produire si, par suite de l'infection, les molécules CMH-II prennent une conformation anormale, soit parce qu'elles s'associent à des partenaires nouveaux, soit parce qu'elles sont vides de peptides antigéniques ou, au contraire, qu'elles contiennent un peptide particulier. Une telle activation facilite- rait l'infection mais également la mort lente des cellules T continuellement activées sans spécificité antigénique.

[1. Haffar OK, et al. Proc Natl Acad Sci USA 1993; 90 : 11094-8.]

\section{Localisation du gène de la myopathie de Fukuyama: 9q31-33.} La dystrophie musculaire congénitale de Fukuyama, dont la transmission est récessive autosomique, est une des myopathies les plus fréquentes au Japon. C'est donc à une équipe japonaise que l'on doit la récente localisation chromosomique $\mathrm{du}$ locus de cette affection [1]. La myopathie de Fukuyama associe un retard mental sévère et une micropolygyrie cérébrale et cérébelleuse (anomalie de la taille et du nombre des circonvolutions, probablement due à un défaut de migration neuronale) à l'atteinte musculaire, essentiellement caractérisée par une hypotonie précoce. Une équipe américaine avait précédemment rapporté la forte diminution d'une des glycoprotéines membranaires associées à la dystrophine (protéine de $43 \mathrm{kDa}$ ) dans le muscle de patients atteints de cette maladie $\left(\mathrm{m} / \mathrm{s} n^{\circ} 6-7\right.$, vol. 9 , p. 814). C'est donc vers la localisation chromosomique de cette protéine en 3p2l qu'ont d'abord été orientées les premières recherches. L'analyse de liaison a porté sur vingt et une familles où ségrégeait une myopathie de Fukuyama et a d'emblée permis d'exclure ce locus. L'observation d'un patient porteur d'une vraisemblable pathologie de contiguilté, associant une maladie de Fukuyama et un Xeroderma pigmento sum de groupe $\mathrm{A}$, dont la localisation en 9q31 était connue, a par la suite permis de rechercher une liaison avec les marqueurs du bras long du chromosome 9. Cette fois, l'hypothèse fut la bonne puisqu'un lod-score significatif a été obtenu pour les marqueurs de la région 9q31-q33 et que ce locus a été trouvé à l'état homozygote chez les patients atteints de myopathie de Fukuyama. Le conseil génétique et le diagnostic pré- natal de cette maladie devraient donc désormais pouvoir être proposés. Néanmoins, l'hypothèse déjà émise $\left(m / s \quad n^{\circ} 6-7\right.$, vol. 9, p. 814) d'une interaction fonctionnelle entre la protéine codée par ce locus et une des protéines du sarcolemme associées à la dystrophine reste toujours en vigueur.

[1. Toda T, et al. Nature Genet 1993; 5: 283-6.]

Les coraux blanchissent sous le soleil des tropiques. Depuis plus de dix ans les barrières de corail tropicales perdent leur couleur du fait de la réduction du nombre de zooxanthelles, l'algue symbiotique qui vit dans les tissus coralliens et contient les pigments photosynthétiques. Ce phénomène, cyclique et de plus en plus fréquent, a été attribué à l'élévation de la température des eaux marines. Les variations de température ne peuvent cependant pas expliquer, à elles seules, la décoloration massive des coraux des Caraïbes en 1987 et 1990. Celle-ci est apparue alors que les conditions climatiques étaient particulièrement clémentes avec une mer très calme et exceptionnellement claire. En l'absence de particules organiques et inorganiques en suspension, les rayons ultraviolets $(280-400 \mathrm{~nm})$ ont pu atteindre les coraux avec une intensité plus forte et à des profondeurs plus importantes. Une large proportion des coraux décolorés des Caraïbes se trouvait à plus de 20 mètres de profondeur. Pour étudier l'effet des radiations ultraviolettes sur les coraux, deux espèces communes de coraux ont été transplantées à différentes profondeurs et exposées aux rayons ultraviolets ambiants ou, au contraire, mises à l'abri de ceux-ci par une protection acrylique [1]. Aucune variation de température de l'eau ne fut enregistrée pendant la durée des expériences. Toutes les colonies coralliennes, transplantées à 12 mètres de profondeur et non protégées des rayons 
ultraviolets, se sont décolorées en 3 semaines. Les colonies n'ayant pas reçu de rayonnement ultraviolet ou celles transplantées à de plus grandes profondeurs ont gardé leur couleur. La décoloration des colonies peut être attribuée à la réduction du nombre de zooxanthelles et de la quantité de chlorophylle par centimètre carré de tissu corallien. Les coraux neutralisent habituellement les effets dévastateurs des UV en synthétisant des composants filtrants apparentés à la mycosporine (MAA : mycosporine like amino acids). Plus les coraux vivent en profondeur, moins ils synthétisent de MAA et plus ils sont sensibles à une augmentation brutale du rayonnement ultraviolet. En effet, en trois semaines, le temps nécessaire à l'obtention d'une décoloration importante, les coraux ne peuvent pas accumuler suffisamment de MAA pour se protéger des rayons ultraviolets. Par des calculs savants, les auteurs de ce travail montrent que les conditions climatiques, à l'époque de la décoloration des coraux des Caraïbes, étaient telles que l'intensité des rayons ultraviolets (essentiellement UVA) était grandement suffisante pour induire cette décoloration à plus de 20 mètres de profondeur. En somme, ce qui fait brunir les uns fait pâlir les autres avec, dans les deux cas, des conséquences malheureuses. [1. Gleason DF, et al. Nature 1993 ; 365 : 836-8.]

\footnotetext{
Mesure du calcium intranucléaire par l'aequorine recombinante ciblée vers le noyau. Des changements de concentration intranucléaire du $\mathrm{Ca}^{2+}$ ont été mis en cause dans des événements nucléaires aussi importants que l'activation de facteurs de transcription de l'ADN, la rupture de la membrane nucléaire, ou l'apoptose. Mais c'était là pure spéculation car aucune méthode jusqu'à présent n'avait permis de mesurer spécifiquement la concentration nucléaire du $\mathrm{Ca}^{2+}$,
}

$\left[\mathrm{Ca}^{2+}\right]_{n}$. Deux équipes italienne et suisse viennent de rapporter les premiers résultats d'une méthode de mesure de $\left[\mathrm{Ca}^{2+}\right] \mathrm{n}$ par l'aquorine recombinante [1]. L'aequorine est une protéine de la méduse $A$ Equorea, formée de deux parties, l'apoprotéine de $22 \mathrm{kDa}$, et un groupement prosthétique, la cœlentérazine, qui émet de la lumière en présence de traces de $\mathrm{Ca}^{2+}$. La transfection de cellules avec l'ADNc de l'aequorine a permis d'exprimer la protéine directement dans le cytoplasme de ces cellules et d'y mesurer la concentration de $\mathrm{Ca}^{2+}$. La fusion d'une autre protéine à la partie $\mathrm{N}$ terminale de l'aquorine ne modifie pas sa réponse au $\mathrm{Ca}^{2+}$, et les mêmes auteurs ont déjà transfecté une construction comportant la séquence de ciblage mitochondrial de la sous-unité VIII de la cytochrome oxydase $c$ humaine fusionnée à l'ADNc de l'aequorine, et vérifié que la protéine chimérique s'exprimait spécifiquement dans les mitochondries où ils ont pu mesurer les variations rapides $\mathrm{du}$ $\mathrm{Ca}^{2+}$ [2]. Une stratégie similaire a été employée ici pour cibler l'aequorine dans le noyau de cellules intactes: une partie de l'ADNc du récepteur des glucocorticoïdes de rat, qui comportait la séquence signal de localisation nucléaire, a été fusionnée à l'ADNc de l'aequorine (seul l'acide aminé N-terminal de l'aequorine manque dans la protéine chimère). La partie de l'ADNc du récepteur des glucocorticoïdes est celle qui code pour les acides aminés 407-524 qui comporte le domaine de liaison à l'ADN et le signal de localisation nucléaire NL1 [3]. Cette partie d'ADNc a déjà été utilisée avec succès pour diriger vers le noyau le gène de la $\beta$ galactosidase. L'ADNc chimérique a été cloné dans un vecteur d'expression et transfecté dans des cellules HeLa avec le plasmide pSV2néo pour pouvoir sélectionner les clones transfectés de façon stable. L'aequorine recombinante s'exprime dans ces cellules, en majeure partie dans le noyau. Enfin on a pu mesurer la
$\left[\mathrm{Ca}^{2+}\right]_{\mathrm{n}}$. Au repos, la concentration nucléaire du $\mathrm{Ca}^{2+}$ est la même que celle du cytoplasme. Après stimulation par l'histamine (agoniste extracellulaire dont l'effet est relayé par l'inositol triphosphate), les variations du $\mathrm{Ca}^{2+}$ nucléaire suivent la même cinétique que celles du $\mathrm{Ca}^{2+}$ cytoplasmique. Le noyau n'a donc pas pas de mécanisme de régulation du $\mathrm{Ca}^{2+}$ indépendant. Cependant, cette stimulation entraîne une élévation de la $\left[\mathrm{Ca}^{2+}\right]_{n}$ supérieure à celle observée dans le cytoplasme. Ce phénomène semble traduire la proximité du noyau des citernes du réticulum endoplasmique qui sont les réservoirs du $\mathrm{Ca}^{2+}$ cellulaire, ou la présence de récepteurs de l'inositol triphosphate sur la membrane nucléaire induisant le relargage du $\mathrm{Ca}^{2+}$ stocké dans l'espace périnucléaire. Dans des cellules à l'architecture aussi complexe que les neurones, l'élévation localisée de la concentration de $\mathrm{Ca}^{2+}$ pourrait affecter de façon variable la $\left[\mathrm{Ca}^{2+}\right]_{n}$ et les événements nucléaires.

[1. Brini M, et al. EMBOJ 1993 ; 12 : 4813-9.]

[2. Rizzuto R, et al. Nature 1992 ; 358 : 325-7.]

[3. Picard D, et al. EMBOJ 1987 ; 6 : 3333-40.]

Un récepteur chimérique fonctionne (presque) comme on l'espérait. Les équipes de Jean-Pierre Changeux (Institut Pasteur, Paris, F) et de Daniel Bertrand (faculté de médecine, Genève, $\mathrm{CH}$ ) continuent leur fructueux travail de dissection moléculaire du récepteur nicotinique de l'acétylcholine. Après avoir montré que la modification de trois acides aminés dans le segment $\mathrm{M}_{2}$ pouvait changer un courant cationique en anionique $\left(\mathrm{m} / \mathrm{s} n^{\circ} 10\right.$, vol. 8 , p. 1118), ils révèlent aujourd'hui [1] l'indépendance fonctionnelle de sous-domaines du récepteur grâce à la confection de chimères entre ce récepteur et l'un de ceux de la sérotonine, le récepteur 5HT3. Ces deux 
récepteurs font partie d'une famille de canaux liés à un ligand possédant quatre domaines transmembranaires $\left(\mathbf{M}_{1}-\mathbf{M}_{4}\right)$ et un long domaine $\mathrm{N}$-terminal extracellulaire contenant le site de liaison du ligand. En associant le domaine $\mathrm{N}$-terminal de la sous-unité $\alpha_{7}$ du récepteur nicotinique neuronal et les domaines transmembranaires du récepteur 5HT3, les auteurs ont obtenu un récepteur fonctionnel liant l'acétylcholine ou la nicotine mais présentant la sélectivité ionique du canal 5HT3. Chacun des deux domaines ( $\mathrm{N}$-terminal et transmembranaire) fonctionne donc d'une façon autonome pour remplir ces rôles. En revanche, les vitesses d'activation et de désensibilisation du canal chimérique se sont révélées intermédiaires entre celles calculées pour les deux récepteurs d'origine. Certaines propriétés des récepteurs-canaux dépendent donc apparemment de façon stricte d'un sous-domaine de la protéine alors que d'autres sont liées sans doute à la structure, tertiaire ou quaternaire, de la molécule entière.

[1. Eiselé JL, et al. Nature 1993; 366 : $479-83$.

Auto-immunité et superantigènes. L'encéphalomyélite allergique expérimentale (EAE) est un modèle animal d'étude de la sclérose en plaques. Certaines lignées de souris immunisées contre la MBP de rat (myelin basic protein) présentent des signes caractéristiques de la démyélinisation, pouvant aller jusqu'à la paralysie. En général, les souris récupèrent et ne font pas d'autres poussées. Chez ces souris (PL/J) les cellules $\mathrm{T}$ encéphalitogènes sont principalement porteuses du segment $\mathrm{V} \beta 8$ comme élément variable de la chaîne $\beta$ du récepteur de l'antigène reconnaissant un épitope dominant de la MBP. Or il se trouve que le superantigène $\mathrm{SEB}$ (entérotoxine $\mathrm{B}$ du staphylocoque) stimule préféren-

lymphocytes $\mathrm{T}$ porteurs du V $\beta 8$. Pour tester l'effet d'un activateur des cellules $T$ sur la réapparition de l'EAE, SEB est injecté aux souris, trois à quatre semaines après l'immunisation contre la MBP, lorsqu'elles ne présentent plus de signes de la maladie [1]. Il en résulte une augmentation tout à fait significative du nombre de rechutes par rapport aux souris témoins. Parfois, certaines souris ne présentent aucun signe clinique de démyélinisation après l'immunisation avec le peptide MBP. Faisant suite à l'injection de SEB, l'exacerbation de la maladie se traduit, dans ce cas, par l'apparition de signes cliniques chez $60 \%$ des souris. D'autres souris développent une EAE après immunisation bien qu'elles n'expriment pas le segment V $\beta 8$. Chez ces souris, l'injection de SEB n'a aucune incidence. L'effet amplificateur de SEB sur la maladie est donc directement lié à la réactivation des cellules T V $\beta 8$. Cela est confirmé par le transfert de clones ou de lignées encéphalitogènes porteurs du V $\beta 8$. Ces cellules, préactivées in vitro par SEB ou activées in vivo par injection du superantigène, provoquent une EAE sévère. Les cellules $\mathrm{V} \beta 8$ non encéphalitogènes ne produisent pas cet effet. La production de TNF $\alpha$, induite par l'activation des cellules $\mathrm{T}$ encéphalitogènes par SEB, semble être un élément décisif de l'exacerbation de la maladie. Ce travail montre qu'un agent infectieux peut influencer l'évolution d'une maladie auto-immune en agissant directement sur les lymphocytes. Schématiquement, la progression par poussées des maladies auto-immunes pourrait résulter de la réactivation répétée de clones auto-immuns au cours de certaines infections. Une action indirecte n'est pas à écarter car des superantigènes n'interagissant pas avec le récepteur de l'antigène des cellules encéphalitogènes peuvent parfois avoir un effet amplificateur sur la maladie [2]. Quoi qu'il en soit, ces travaux montrent à nouveau que l'activation directe, ou par cytokines interposées, des cellules auto-immunes est la résultante de nombreux facteurs et mécanismes dont seule la complexité n'est plus mystérieuse.

[1. Brocke S, et al. Nature 1993; 365 : 642-4.]

[2. Schiffenbauer J, et al. Proc Natl Acad Sci USA 1993; 90 : 8543-6.]

La protéine CD34 endothéliale lie la L-sélectine des leucocytes. Les interactions d'adhérence entre la L-sélectine des leucocytes et l'endothélium permettent la migration des lymphocytes au travers des ganglions lymphatiques et la migration des polynucléaires neutrophiles vers les sites inflammatoires [1]. Une équipe de Genentech (San Francisco, CA, USA) et de l'université de Californie à San Francisco vient de montrer que la glycoprotéine sulfatée impliquée dans ces interactions au niveau de l'endothélium cubique des veinules post-capillaires [1] des ganglions lymphatiques, isolée récemment et nommée Sgp90 [2], n'est autre que la protéine CD34, la sialomucoprotéine exprimée sur les cellules souches hématopoiétiques [3, 4] et les endothéliums [5]. La L-sélectine des polynucléaires neutrophiles est nécessaire à l'étape initiale de mobilisation, préalable à l'adhérence relayée par les intégrines et la diapédèse des neutrophiles au cours de l'inflammation. Bien que ces auteurs n'aient pas montré la liaison de la L-sélectine à la CD34 vasculaire en dehors des organes lyınphoïdes, la large distribution endothéliale de celle-ci et la démonstration de son rôle de ligand majeur dans les ganglions lymphatiques laissent à penser qu'elle a aussi une fonction dans la mobilisation des leucocytes lorsqu'elle est glycosylée de façon adéquate. La régulation de sa fonction comporte une glycosylation variable, peut-être sélective du vaisseau considéré, sa translocation et son oligomérisation éventuelle à la surface de la cellule 
endothéliale : la glycosylation spécifique du vaisseau pourrait expliquer pourquoi les leucocytes n'adhèrent qu'aux veinules post-capillaires, alors que CD34 est exprimée à la fois sur les capillaires et les veinules. La forme de $\mathrm{CD} 34$ qui s'exprime sur les cellules souches hématopoïétiques a-t-elle une glycosylation particulière qui lui permettrait de se lier à une lectine du stroma de la moelle osseuse?

[1. Imai Y, et al. Nature 1993; 361 : 555.]

[2. Néel D, et al. médecine/sciences $1992 ; 8: 233-8$.]

[3. Blanchet JP, et al. médecine/sciences $1993 ; 9$ : 959-63.]

[4. Hatzfeld J, et al. médecine/sciences $1993 ; 9$ : 1110-2.]

[5. Baumhueter S, et al. Science $1993 ; 262: 436-8$.]

apoptose des lymphocytes B au repos induite par le complexe majeur d'histocompatibilité. Les molécules de classe II du complexe majeur d'histocompatibilité (CMHII), qui présentent les antigènes peptidiques aux lymphocytes $\mathrm{T} \mathrm{CD}^{+}$ auxiliaires, sont aussi des récepteurs capables de transmettre des signaux aux lymphocytes B qui les expriment. Le contact entre lymphocytes $\mathrm{T}$ et $\mathrm{B}$ reconnaissant un même antigène (natif ou sous forme peptidique) se traduit par l'activation et la différenciation des deux populations cellulaires sous l'impulsion de signaux transmis par des récepteurs membranaires (comme, par exemple, le récepteur de l'antigène des lymphocytes T (TcR) et le CMH-II des lymphocytes B). L'engagement du CMH-II dans une interaction moléculaire peut cependant avoir des effets différents selon le stade de maturation des lymphocytes B [1]. Ainsi, la fixation d'anticorps monoclonaux anti-CMH-II, à la surface des lymphocytes $B$ au repos, bloque la multiplication et l'activation cellulaires en induisant une élévation d'AMPc et la translocation de la $\mathrm{m} / \mathrm{s} n^{\circ}$ I vol. 10, janvier 94
PKC du cytosol vers le noyau, sans modifier la concentration en calcium intracellulaire ou le métabolisme des inositol-phosphates. De plus, le traitement des lymphocytes B au repos avec des anticorps antiCMH-II ou d'autres agents augmentant la concentration en AMPc provoque leur mort par apoptose. $\mathrm{Ni}$ l'augmentation de l'AMPc, ni l'apoptose ne sont observées si les cellules sont préalablement activées avec des anticorps anti-immunoglobuline et de l'interleukine 4. Lors du contact entre les lymphocytes $\mathrm{T}$ et $\mathrm{B}$, l'interaction TCR/CMH-II aurait donc deux effets opposés : la multiplication des lymphocytes $B$ mûrs et l'élimination des lymphocytes B immatures. Ce mécanisme permettrait l'établissement d'une réponse humorale spécifique d'un antigène, car seuls les lymphocytes B ayant préalablement reconnu un antigène par l'intermédiaire de leurs immunoglobulines de surface pourraient se multiplier au contact des lymphocytes T. Par ce biais, l'expansion des lymphocytes B non spécifiques d'un antigène serait évitée.

[1. Newell MK, et al. Proc Natl Acad Sci USA 1993; 90 : 10459-63.]

\section{- Reconnaissance de l'antigène} et activation de $\mathrm{p} 21^{\text {ras }}$ des lymphocytes $\mathrm{B}$. La signalisation transmembranaire provoquée par la fixation d'anticorps anti-immunoglobulines à la surface des lymphocytes B fait intervenir des voies d'activation auxquelles les protéines $G$ ne semblent pas étrangères. La participation des petites protéines $\mathrm{G}$ dans ce processus est documentée et il est possible, par exemple, que p21 ras s'associe aux immunoglobulines de surface dans certaines conditions. L'activité de p21 ras peut être estimée par les quantités relatives de sa forme active (associée au GTP) et de sa forme inactive (associée au GDP). L'état d'activation de $\mathrm{p} 21^{\text {ras }}$ dépend donc de son activité GTPa- sique intrinsèque, une fonction qui est réglée par rasGAP (ras GTPase activating protein) $\left(\mathrm{m} / \mathrm{s}, n^{\circ} 5, v o l .8\right.$, p. 471). L'activité de rasGAP dépend, quant à elle, de son état de phosphorylation qui peut être modulé par l'interaction des antiimmunoglobulines à la surface des lymphocytes B. Ce travail précise l'intervention de $\mathrm{p} 2 \mathrm{l}^{\text {ras }}$ et rasGAP dans l'activation cellulaire induite par la reconnaissance d'un antigène par les immunoglobulines de surface [1]. La cellule utilisée provient d'un lymphome murin et reconnaît le TNP par l'intermédiaire de ses IgM membranaires. Après une courte stimulation antigénique, le rapport forme active/forme inactive de p21 ras s'accroît fortement, sans que soit modifié le taux d'échange des nucléotides. L'activité enzymatique de rasGAP est inhibée par ce même traitement pendant au moins vingt minutes, avec un maximum à une minute. La phosphorylation transitoire des tyrosines pourrait être une étape précoce de l'inactivation de $\operatorname{rasGAP}\left(\mathrm{m} / \mathrm{s}, n^{\circ} 10\right.$, vol. 8 , p. 1095). Il a été rapporté que l'activité de rasGAP peut être inhibée par son association avec la protéine p190. Cela ne semble pas être le cas ici car le complexe p190/rasGAP n'a pu être détecté dans ces cellules, proches du phénotype mûr des lymphocytes B IgM $^{+}$. Cette observation permet de penser que le mécanisme d'inactivation de rasGAP, par une stimulation antigénique, pourrait être lié au stade de différenciation des lymphocytes $\mathrm{B}$.

[1. Lazarus AH, et al. J Exp Med $1993 ; 178: 1765-9$.

Des régions du cortex cérébral sont déjà spécifiées dans la couche germinative. Les neurones corticaux naissent, au cours de la vie intra-utérine, de la prolifération de précurseurs situés dans une couche germinative péri-ventriculaire que l'on appelle la plaque corticale. Après la neurogenèse et la migration, ils forment des aires corticales (voir $\mathrm{m} / \mathrm{s}$, Lexique de neurobiologie,

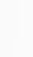


1992) dont les caractéristiques cytologiques et architecturales diffèrent. Il n'avait pas jusque-là été possible de distinguer entre elles les cellules de la plaque corticale et l'on ne savait pas si les neurones corticaux naissaient d'une plaque uniforme ou s'il existait, dès ce niveau, une spécification régionale de cellules souches possédant des propriétés particulières. Le groupe d'Henri Kennedy (Inserm, Lyon France) vient d'apporter un élément important en faveur de cette dernière hypothèse [1]. Le cortex visuel du primate adulte contient des aires qui different par leur densité neuronale, deux fois plus forte dans l'aire striée que dans les autres. Les auteurs ont recherché l'origine de cette hyperdensité cellulaire en injectant chez des foetus de la thymidine tritiée qui, en s'intégrant dans l'ADN de cellules en phase $S$ (synthèse d'ADN), a révélé celles impliquées dans le cycle cellulaire au moment précis de l'injection. Deux résultats auraient pu être obtenus selon que la plus grande densité neuronale soit liée ou non à une spécification régionale de la plaque corticale: dans le premier cas, cette spécification précoce pourrait se manifester par une différence entre les rythmes de prolifération de la zone donnant naissance à l'aire striée et aux autres, alors qu'une telle différence serait absente dans le second cas. C'est effectivement cette observation qu'ont faite les auteurs qui indiquent, par ailleurs, que l'augmentation du rythme de prolifération n'a lieu que durant un bref laps de temps de la vie foetale et qu'elle est associée vraisemblablement à un raccourcissement de la durée du cycle prolifératif. Des cellules de la plaque corticale possèdent donc déjà des propriétés différentes de celles de leurs voisines et définissent ainsi certaines caractéristiques distinctives d'une aire corticale.

[1. Dehay C, et al. Nature 1993; 366 : 464-6.]
- Un nouveau récepteur de l'antigène spécifique des cellules $\mathrm{T}$ immatures. La maturation des thymocytes dépend en partie de l'interaction du récepteur de l'antigène $(T C R \alpha \beta)$ avec les molécules du complexe majeur d'histocompatibilité. L'expression de la chaîne $\beta$ du récepteur de l'antigène (TCR $\beta$ ) précède, dans le temps, celle de la chaîne $\alpha$. La protéine TCR $\beta$ joue aussi un rôle fondamental dans le développement des cellules T, comme cela a été montré à partir des souris SCID, RAG-/- ou TCR $\alpha$-/-transgéniques pour la chaîne TCR $\beta$. Dans les cellules T matures, la sous-unité TCR $\beta$, non associée à $\operatorname{TCR} \alpha$, est retenue et dégradée dans le réticulum endoplasmique, alors qu'elle est présente à la surface des cellules $\mathrm{T}$ immatures TCR $\alpha-/-$. Le devenir de la protéine TCR $\beta$ dépend donc du stade de différenciation des cellules $T$ qui l'expriment. L'idée généralement acceptée, en l'absence de preuve du contraire, est que les cellules T immatures expriment à leur surface un homodimère TCR $\beta$ fonctionnel, pouvant induire une mobilisation calcique intracellulaire. Cela doit maintenant être reconsidéré à la lumière des travaux récents [1] qui n'ont pas pu mettre en évidence l'existence d'un tel homodimère mais, en revanche, démontrent clairement que TCR $\beta$ est associé à une protéine de $33 \mathrm{kDa}$ (gp33), dont l'expression est spécifique des cellules $\mathrm{T}$ immatures. Après marquage métabolique avec de la leucine tritiée et biotinylation des molécules de surface, gp33 est détectée dans les immunoprécipitations de la sousunité TCR $\beta$ avec laquelle elle forme des ponts disulfures. En s'associant à gp $33, T C R \beta$ échappe donc à une dégradation précoce. Nul doute que le ligand naturel, ou accidentel (superantigène), du complexe TCR $\beta$-gp33 ne tardera pas à être identifié et que les souris déficientes en gp33 seront produites par recombinaison homologue dès que le gène aura été cloné.

[1. Groettrup M, et al. Cell 1993; 75: 283-94.]

Obésité chez des souris transgéniques avec augmentation du transport de glucose dans les adipocytes. Le transporteur de glucose GLUT-4, présent dans les cellules musculaires et les adipocytes, est contrôlé par l'insuline à différents niveaux. L'augmentation de la consommation périphérique de glucose sous l'action de l'insuline passe donc principalement par une activation de ce transporteur. Des chercheurs de Boston (MA, USA) [1] ont créé des souris transgéniques dans lesquelles le gène GLUT-4 a été mis sous le contrôle de séquences régulatrices en ciblant l'expression dans les cellules adipocytaires. Les animaux transgéniques synthétisent des quantités très augmentées de la protéine GLUT-4 et ont un transport de glucose, en l'absence ou en présence d'une stimulation par l'insuline, nettement plus élevé que des animaux témoins de même âge. Ces animaux ont une augmentation de deux à trois fois des lipides corporels totaux, sans aucune modification de la taille des adipocytes, c'està-dire de leur teneur individuelle en triglycérides: ce qui est augmenté, c'est le nombre des adipocytes. Ainsi, l'augmentation du transport de glucose induit-elle une hyperplasie adipocytaire, à l'origine d'un modèle d'obésité. Cela suggère que l'augmentation de l'expression du gène GLUT-4 dans le tissu adipeux stimule la division des préadipocytes ou leur différenciation en adipocytes.

[1. Shepherd BR, et al. J Biol Chem $1993 ; 268$ : 22243-6] 
La levure mérite bien son titre de "mammifère d'honneur"! Observé à l'aide de techniques de microscopie électronique tridimensionnelle, l'appareil de Golgi des cellules eucaryotes se présente comme un ruban continu, avec des diverticules et des anastomoses, dont le tout s'assemble en un réseau très élaboré. En section transverse selon un axe cis-trans, ce ruban peut être subdivisé en trois compartiments. Le premier d'entre eux est constitué d'un réseau de tubules membranaires, appelé le réseau tubulaire-cis. Le second, ou compartiment intermédiaire, se compose de plusieurs éléments empilés (généralement deux à quatre), mais qui sont disposés en continuité le long du ruban golgien. Ce ruban présente des zones relativement peu fenestrées et aplaties (les saccules) qui alternent avec des zones largement perforées, que l'on nomme zones tubulaires intersacculaires. Enfin, le troisième compartiment, ou compartiment trans, se compose de deux ou trois éléments sacculo-tubulaires, se séparant par endroit du ruban golgien et qui semblent progressivement se détacher de l'ensemble. C'est dans ce dernier compartiment que s'effectue la ségrégation des glycoprotéines, et, dans le cas des cellules glandulaires, la formation de grains de sécrétion. Chez la levure Saccharomyces cerevisiae, on retrouve un organite semblable, accomplissant les mêmes fonctions. Chez certains mutants de ces organismes unicellulaires, le transfert intracellulaire des protéines peut être bloqué par des températures non permissives, à différentes étapes de leur parcours telles que le réticulum endoplasmique, l'appareil de Golgi ou les grains de sécrétion, où l'on constate une accumulation des protéines. Faute de méthodes de coloration appropriées, l'examen de ces processus par des techniques de microscopie électronique n'avait pu être effectué jusqu'ici. Deux équipes du CEA, en collaboration avec un laboratoire canadien de l'université McGill, viennent de réaliser, à Saclay
(France), une étude en microscopie électronique tridimensionnelle de l'appareil de Golgi d'un mutant de la levure (sec 7)[1]. Chez le type sauvage, les éléments du Golgi se présentent comme des réseaux isolés de tubules membranaires, largement dispersés dans le cytoplasme. Dans ces réseaux, on trouve des zones qui semblent distendues, remplies d'un matériel coloré, dont la taille se rapproche de celle des grains de sécrétion avoisinants, suggérant que la formation de ces derniers résulte de la fragmentation des éléments du Golgi. Chez les mutants $\sec 7$ maintenus à $37^{\circ} \mathrm{C}$, dans un milieu pauvre en glucose, le nombre des granules diminue progressivement. Parallèlement, les réseaux de tubules golgiens gagnent en complexité et en taille, perdent leurs zones de distension et se transforment progressivement en saccules aplatis, formant des empilements de sept ou huit éléments. Fait inattendu : ces empilements de saccules ressemblent par bien des aspects à ceux que l'on décrit dans l'appareil de Golgi des cellules de mammifères. Toutefois, à la différence de ce dernier, dans le Golgi de ce mutant, on peut voir des connexions évidentes entre les saccules, mais, sauf exception, on ne voit aucune vésicule associée. Lorsque le mutant est soumis à nouveau à une température permissive, les grains de sécrétion réapparaissent, les empilements de saccules disparaissent et peu à peu les réseaux de tubules initiaux se reconstituent. En conjuguant la microscopie électronique tridimensionnelle et une méthode de coloration originale, avec l'usage raisonné de mutants de $S$. cerevisiae affectés dans la sécrétion, il a donc été possible de décrire pour la première fois l'ultrastructure de l'appareil de Golgi normal de la levure, et de montrer comment se forment les granules de sécrétion à partir de ce même appareil.

[1. Rambourg A, et al. Anat Rec 1993 ; 237 : 441-52.] 